\title{
The high-cost, type 2 diabetes mellitus patient: an analysis of managed care administrative data
}

\author{
Juliana L Meyers ${ }^{1 * \dagger}$, Shreekant Parasuraman ${ }^{2 \dagger}$, Kelly F Bell ${ }^{3 \dagger}$, John P Graham ${ }^{3 \dagger}$ and Sean D Candrilli ${ }^{1+}$
}

\begin{abstract}
Background: Type 2 diabetes mellitus (T2DM) affects 25.8 million individuals in the United States and exerts a substantial economic burden on patients, health care systems, and society. Few studies have categorized costs and resource use at the patient level. The goals of this study were to assess predictors of being a high-cost (HC) patient and compare HC T2DM patients with not high-cost (NHC) T2DM patients.

Methods: Using managed care administrative claims data, patients with two or more T2DM diagnoses between 2005 and 2010 were selected. Patients were followed for 1 year after their first observed T2DM diagnosis; patients not continuously enrolled during this period were excluded from the study. Study measures included annual health care expenditures by component (i.e., inpatient, outpatient, pharmacy, total). Patients accruing total costs in the top $10 \%$ of the overall cost distribution (i.e., patients with costs $>\$ 20,528$ ) were classified as $\mathrm{HC}$ a priori; all other patients were considered NHC. To assess predictors of being HC, a logistic regression model was estimated, accounting for demographics; underlying comorbidity burden (using the Charlson Comorbidity Index [CCI] score); diagnoses of renal impairment, obesity, or hypertension; and receipt of insulin, oral antidiabetics only, or no antidiabetics.

Results: A total of 1,720,041 patients met the inclusion criteria; 172,004 were HC. The mean (SD) CCl score for HC patients was 4.3 (3.0) versus 2.1 (1.7) for NHC patients. Mean (SD; upper 95\% confidence interval-lower 95\% confidence interval) annual per-patient costs were $\$ 56,468(\$ 65,604 ; \$ 56,778-\$ 56,157)$ among HC patients and $\$ 4,674$ ( $\$ 4,504$; $\$ 4,695-\$ 4,652)$ among NHC patients. Inpatient care and pharmacy costs were higher for HC patients than for NHC patients. The strongest predictor of being an $\mathrm{HC}$ patient was having a $\mathrm{CCl}$ score of 2 or greater (odds ratio $[O R]=4.896)$, followed by a diagnosis of obesity $(O R=2.106)$, renal impairment $(O R=2.368)$, and insulin use $(\mathrm{OR}=2.098)$.
\end{abstract}

Conclusions: High-cost T2DM patients accrue approximately $\$ 52,000$ more in total annual health care costs than not high-cost T2DM patients. Patients were significantly more likely to be high-cost if they had comorbid conditions, a diagnosis of obesity, or used insulin.

Keywords: Diabetes mellitus, type 2, Health care costs, Economics

\section{Background}

Diabetes is a chronic, progressive condition that, if not managed properly, can lead to numerous health complications and disability. It has been estimated that approximately 25.8 million people in the United States (US) have diabetes [1]. In adults, $90 \%$ to $95 \%$ of patients with diabetes have type 2 diabetes mellitus (T2DM), which is characterized by insulin resistance, pancreatic beta-cell

\footnotetext{
* Correspondence: jmeyers@rti.org

${ }^{\dagger}$ Equal contributors

${ }^{1}$ RTI Health Solutions, 200 Park Offices Drive, Research Triangle Park, NC 27709, USA

Full list of author information is available at the end of the article
}

dysfunction, and excessive glucose production by the liver. Over the next 40 years, the total prevalence of diabetes in the US is expected to more than double, from $5.6 \%$ in 2005 to $12.0 \%$ in 2050 [2].

Treatment of T2DM consists of significant lifestyle adjustments and drug therapy, including oral antidiabetic agents and insulin therapy [3]. The American Diabetes Association and the European Association for the Study of Diabetes have agreed that early intervention with metformin should be used in patients with hyperglycemia to help maintain recommended levels of glycemic control [4]. Because T2DM is a progressive
C Biomed Central

(C) 2014 Meyers et al.; licensee BioMed Central Ltd. This is an Open Access article distributed under the terms of the Creative Commons Attribution License (http://creativecommons.org/licenses/by/2.0), which permits unrestricted use, distribution, and reproduction in any medium, provided the original work is properly credited. 
disease, timely augmentation of therapy with additional agents, such as insulin, sulfonylureas, thiazolidinediones, dipeptidyl peptidase-4 inhibitors, and glucagon like peptide-1 receptor agonists, also is recommended [5].

It is well established that the costs attributable to diabetes (including T2DM) are substantial. One recent estimate suggested that the total economic burden of all types of diabetes in the US exceeds $\$ 174$ billion annually, which includes $\$ 116$ billion in excess medical expenditures and $\$ 58$ billion in reduced nation productivity [6]. Medical costs attributed to all types of diabetes include $\$ 27$ billion for direct care, $\$ 58$ billion to treat patients with diabetes-related chronic complications, and $\$ 31$ billion in excess general medical costs [6].

Significant evidence exists showing the relationship between diabetes-related costs and observed glucose values [7-12]. However, as informative as these studies have been in communicating the economic impact of diabetes, many studies have included data from sources outside the US or did not focus on managed care populations. Further, identifying T2DM patients who could be considered high cost $(\mathrm{HC})$ is of significant interest to health care payers, given the rising health care costs in the US-interventions could be developed that would focus on patients who are likely to become $\mathrm{HC}$ and therefore minimize costs of the disease. Previous studies have identified $\mathrm{HC}$ patients in other disease areas (e.g., acute coronary syndromes) [13]; but to our knowledge, no retrospective analysis has been published that examines $\mathrm{HC}$ patients with T2DM.

The goal of this analysis was to document the actual health care costs incurred by payers for a T2DM population and to determine which factors were associated with patients in the higher tiers of the T2DM cost distribution. Additionally, this study compared health care costs for patients who were identified as $\mathrm{HC}$ with patients who were identified as not high cost (NHC).

\section{Methods}

This analysis used the LifeLink database (formerly PharMetrics), a commercially available source of computerized administrative claims from 95 managed care health plans covering more than 61 million unique patients. The database included claims from private health plans in all four US geographic regions and had an age and sex distribution representative of national managed care enrollment. The database included patient-level demographics and periods of health plan enrollment; primary and nonprimary diagnoses; detailed information about hospitalizations, diagnostic testing, and therapeutic procedures; inpatient and outpatient physician services; prescription drug use; and cost data in the form of managed-care reimbursement rates for each service. Data are tracked longitudinally within patients via de-identified and unique patient numbers. For the purposes of this analysis, the most recent 5 years of data were used. RTI International's institutional review board determined that this study met all criteria for exemption. Data were available from January 1, 2005, through December 31, 2010.

All patients with at least two diagnoses of T2DM (International Classification of Diseases, Ninth Revision, Clinical Modification [ICD-9-CM] codes $250 \times \times 0$ and $250 . \times 2$ ) during the selection window (January 1, 2005, through December 31, 2009) were initially identified for study inclusion. Requiring two T2DM diagnoses helped to rule out patients who may have been suspected of having T2DM but were never formally diagnosed and likely eliminated patients who were miscoded as having T2DM. For each patient identified, his or her index date was defined as the date of the first observed T2DM diagnosis. To allow for adequate follow-up time to address the research questions and to ensure that any observed lack of health care events was the result of no medical activity and not the result of cessation of care, patients were required to have at least 12 months of continuous post-index date observation. Furthermore, to obtain the largest possible sample size of patients with T2DM, we did not require patients to have received a diabetic medication, nor was any pre-index date enrollment required.

Subgroups of patients in the T2DM population were identified as $\mathrm{HC}$ or $\mathrm{NHC}$ on the basis of where their annual costs fell within the overall T2DM cost distribution. We identified two subgroups of HC T2DM patients a priori: those patients whose costs were in the 90th or greater percentile of all-cause costs and those patients whose costs were in the 80th or greater percentile of allcause costs. We also identified two subgroups of NHC T2DM patients: those patients whose costs were below the 90th percentile of all-cause costs and those patients whose costs were below the 80th percentile of all-cause costs. We chose to assess both the top 10\% and top 20\% of patients in the all-cause cost distribution for several reasons. First, it is a well-regarded rule of thumb that the top $20 \%$ of patients accrue $80 \%$ of health care costs [14]. We wished to evaluate whether this rule held true in a T2DM population. Second, previous studies examining $\mathrm{HC}$ patients in other disease areas have used similar methodology [13]. We also felt that it was important to see if patient characteristics changed as we went from the top $20 \%$ of patients to the top $10 \%$ of patients. For this analysis examining $\mathrm{HC}$ and $\mathrm{NHC}$ T2DM patients, all-cause costs were examined (rather than T2DM-related costs) because many patients likely had coexisting conditions (e.g., hypertension or renal impairment) that were T2DM related but that may not have been coded as such in the claims.

$\mathrm{HC}$ patients then were compared with NHC patients. Key outcome measures that were analyzed included 
patient characteristics, overall (all-cause) health care resource use and costs, and T2DM-related resource use and costs. All outcome measures presented in this paper were assessed over a 1-year post-index date follow-up period, unless stated otherwise. Patient characteristics that were assessed at the index date included age, sex, geographic region, insurance payer type (i.e., commercial, Medicare, Medicaid, self, Medicare Gap, and missing/unknown), and health plan type (i.e., health maintenance organization, preferred provider organization, point of service, indemnity, and missing/unknown). To assess overall comorbidity burden, we calculated a Charlson Comorbidity Index (CCI) score for each patient over the 1-year follow-up period. The mean CCI score, along with the number and percentage of patients with a score $<2$ or $\geq 2$, was reported. The CCI score included 17 categories of comorbidities, as defined by ICD-9-CM diagnosis and procedure codes, with associated weights corresponding to the severity of the comorbid condition of interest [15]. Because all patients in the study had a diagnosis of T2DM and because we wished to evaluate underlying comorbidity burden independent of T2DM, comorbidities corresponding to T2DM were removed from the CCI score (i.e., we did not want the CCI to be inflated for all patients). Antidiabetic agents received were reported by class (i.e., sulfonylureas, meglitinide, biguanide, thiazolidinedione, alpha-glucosidase inhibitor, dipeptidyl peptidase-4, glucagon-like peptide-1, and insulin).

For each patient, overall health care utilization and associated costs were aggregated across all encounters, regardless of reason, observed during the 1 -year postindex date period. The following categories of overall health care utilization and costs were evaluated and reported: inpatient, skilled nursing facility, emergency department, outpatient hospital, office visit, laboratory service, other outpatient care, pharmacy, and total health care utilization. For each category of overall health care utilization, the number and percentage of patients with a visit or admission, the mean (standard deviation [SD]) number of visits or admissions, and the per-patient costs were reported. Additionally, for patients with an inpatient or skilled nursing facility admission, the average number of days per admission was reported.

The total volume and associated costs of health care services specifically related to T2DM also was reported. Hospital admissions related to T2DM were identified by searching for inpatient hospital confinements in which T2DM was recorded as the primary discharge diagnosis (i.e., ICD-9-CM codes $250 \times 0$ and $250 . \times 2$ ). Office, emergency department, outpatient hospital, other outpatient visits, and laboratory services related to T2DM were identified by searching for medical claims with any diagnosis (i.e., primary or secondary) of T2DM and a relevant place-of-service code for the care setting of interest.
Additionally, we evaluated the use and associated costs of all disease-specific medications for which a claim was generated. Medications were identified using appropriate National Drug Codes and Healthcare Common Procedure Coding System codes, brand and generic product names, and therapeutic class descriptions provided in the database.

All analyses were carried out using SAS (Version 9; Cary, North Carolina) statistical software. Descriptive analyses entailed the tabular display of mean and SDs for continuous variables of interest (e.g., total health care costs) and the frequency distribution of categorical variables of interest (e.g., health plan type). All-cause and diabetes-related health care costs were updated to 2011 US dollars, using the medical care component of the US Consumer Price Index.

Logistic regression models were estimated to assess predictors of being an $\mathrm{HC}$ patient with T2DM (separate models for the top-10\% and the top- $20 \%$ groups). The dependent variable was a dichotomous (i.e., 0 or 1 ) variable indicating whether the patient was in the $\mathrm{HC}$ cohort. Demographic characteristics that have repeatedly been shown to be associated with costs were used as independent variables and included patient age (i.e., < 35 years, $35-44$ years, and $45-54$ years vs. $\geq 55$ years), sex (i.e., male vs. female), geographic region (i.e., South, Midwest, and West vs. East), health plan type (i.e., preferred provider organization, point of service, indemnity, and missing/unknown vs. health maintenance organization), and payer type (i.e., Medicaid, Medicare, self, Medicare Gap, and missing/unknown vs. commercial). Clinical variables available in the database were also selected as independent variables and included the CCI score (i.e., CCI score $<2$ vs. CCI score $\geq 2$ ), the types of pharmacologic treatments the patient received (i.e., insulin and oral antidiabetic medications vs. no pharmacological treatment), a diagnosis of renal impairment (i.e., had a renal impairment diagnosis vs. did not have a renal impairment diagnosis), and a diagnosis of obesity (i.e., had an obesity diagnosis vs. did not have an obesity diagnosis). Patients with missing age, sex, health plan, and health payer information were excluded from the regression models.

\section{Results}

Among the 1.72 million T2DM patients in the database who met the initial study inclusion and exclusion criteria, 344,019 were identified as being in the top $20 \%$ of the cost distribution (i.e., costs $>\$ 10,901$ ), and 172,004 were identified as being in the top $10 \%$ of the cost distribution (i.e., costs $>\$ 20,528$ ) (Table 1). Mean (SD) patient age among patients in the top $20 \%$ of the cost distribution was 57.2 (13.7) years versus 57.7 (14.9) years among patients in the bottom $80 \%$ of the cost distribution. In both the top $20 \%$ and the bottom $80 \%$ of patients, sex 
Table 1 Characteristics of the study sample, by cohort

\begin{tabular}{|c|c|c|c|c|c|c|c|c|}
\hline & \multicolumn{8}{|l|}{ Cohort } \\
\hline & \multicolumn{2}{|c|}{ Top $10 \%$ of costs } & \multicolumn{2}{|c|}{ Bottom $90 \%$ of costs } & \multicolumn{2}{|c|}{ Top $20 \%$ of costs } & \multicolumn{2}{|c|}{ Bottom $80 \%$ of costs } \\
\hline & $\mathrm{N}$ & $\%$ & $\mathrm{~N}$ & $\%$ & $\mathrm{~N}$ & $\%$ & $\mathbf{N}$ & $\%$ \\
\hline Total sample & 172,004 & & $1,548,037$ & & 344,019 & & $1,376,022$ & \\
\hline \multicolumn{9}{|l|}{ Age (years) } \\
\hline$<18$ & 1,194 & $0.69 \%$ & 17,232 & $1.11 \%$ & 3,469 & $1.01 \%$ & 14,957 & $1.09 \%$ \\
\hline $18-25$ & 1,445 & $0.84 \%$ & 17,163 & $1.11 \%$ & 3,269 & $0.95 \%$ & 15,339 & $1.11 \%$ \\
\hline $25-34$ & 4,920 & $2.86 \%$ & 56,918 & $3.68 \%$ & 11,866 & $3.45 \%$ & 49,972 & $3.63 \%$ \\
\hline $35-44$ & 14,922 & $8.68 \%$ & 164,584 & $10.63 \%$ & 31,878 & $9.27 \%$ & 147,628 & $10.73 \%$ \\
\hline $45-54$ & 40,506 & $23.55 \%$ & 359,704 & $23.24 \%$ & 80,397 & $23.37 \%$ & 319,813 & $23.24 \%$ \\
\hline $55-64$ & 69,775 & $40.57 \%$ & 474,253 & $30.64 \%$ & 130,935 & $38.06 \%$ & 413,093 & $30.02 \%$ \\
\hline$\geq 65$ & 39,168 & $22.77 \%$ & 457,026 & $29.52 \%$ & 82,028 & $23.84 \%$ & 414,166 & $30.10 \%$ \\
\hline Missing/unknown & 74 & $0.04 \%$ & 1,157 & $0.07 \%$ & 177 & $0.05 \%$ & 1,054 & $0.08 \%$ \\
\hline Mean (SD) & 57.46 & $(12.89)$ & 57.61 & $(14.82)$ & 57.17 & $(13.66)$ & 57.7 & $(14.87)$ \\
\hline \multicolumn{9}{|l|}{ Sex } \\
\hline Male & 86,359 & $50.21 \%$ & 789,524 & $51 \%$ & 164,587 & $47.84 \%$ & 711,296 & $51.69 \%$ \\
\hline Female & 85,630 & $49.78 \%$ & 758,425 & $48.99 \%$ & 179,402 & $52.15 \%$ & 664,653 & $48.30 \%$ \\
\hline Missing/unknown & 15 & $0.01 \%$ & 88 & $0.01 \%$ & 30 & $0.01 \%$ & 73 & $0.01 \%$ \\
\hline \multicolumn{9}{|l|}{ Geographic region } \\
\hline East & 55,618 & $32.34 \%$ & 521,500 & $33.69 \%$ & 113,485 & $32.99 \%$ & 463,633 & $33.69 \%$ \\
\hline South & 36,130 & $21.01 \%$ & 371,487 & $24 \%$ & 71,733 & $20.85 \%$ & 335,884 & $24.41 \%$ \\
\hline Midwest & 55,462 & $32.24 \%$ & 444,249 & $28.70 \%$ & 110,809 & $32.21 \%$ & 388,902 & $28.26 \%$ \\
\hline West & 24,794 & $14.41 \%$ & 210,801 & $13.62 \%$ & 47,992 & $13.95 \%$ & 187,603 & $13.63 \%$ \\
\hline \multicolumn{9}{|l|}{ Health plan type } \\
\hline Health maintenance organization & 37,332 & $21.70 \%$ & 293,868 & $18.98 \%$ & 73,580 & $21.39 \%$ & 257,620 & $18.72 \%$ \\
\hline Preferred provider organization & 96,019 & $55.82 \%$ & 873,018 & $56.40 \%$ & 190,440 & $55.36 \%$ & 778,597 & $56.58 \%$ \\
\hline Point of service & 23,776 & $13.82 \%$ & 178,510 & $11.53 \%$ & 45,378 & $13.19 \%$ & 156,908 & $11.40 \%$ \\
\hline Indemnity & 13,218 & $7.68 \%$ & 181,335 & $11.71 \%$ & 31,063 & $9.03 \%$ & 163,490 & $11.88 \%$ \\
\hline Missing/unknown & 1,659 & $0.96 \%$ & 21,306 & $1.38 \%$ & 3,558 & $1.03 \%$ & 19,407 & $1.41 \%$ \\
\hline \multicolumn{9}{|l|}{ Payer type } \\
\hline Commercial & 125,980 & $73.24 \%$ & $1,156,587$ & $74.71 \%$ & 253,795 & $73.77 \%$ & $1,028,772$ & $74.76 \%$ \\
\hline Medicaid & 3,154 & $1.83 \%$ & 17,382 & $1.12 \%$ & 6,019 & $1.75 \%$ & 14,517 & $1.05 \%$ \\
\hline Medicare & 18,213 & $10.59 \%$ & 91,170 & $5.89 \%$ & 31,044 & $9.02 \%$ & 78,339 & $5.69 \%$ \\
\hline Self & 19,946 & $11.60 \%$ & 213,595 & $13.80 \%$ & 42,432 & $12.33 \%$ & 191,109 & $13.89 \%$ \\
\hline Medicare Gap & 3,987 & $2.32 \%$ & 60,944 & $3.94 \%$ & 9,317 & $2.71 \%$ & 55,614 & $4.04 \%$ \\
\hline Missing/unknown & 724 & $0.42 \%$ & 8,359 & $0.54 \%$ & 1,412 & $0.41 \%$ & 7,671 & $0.56 \%$ \\
\hline \multicolumn{9}{|l|}{ CCI Score ${ }^{a}$} \\
\hline Mean (SD) & $4.27(3.04)$ & & $2.07(1.68)$ & & $3.66(2.75)$ & & $1.95(1.55)$ & \\
\hline $\mathrm{CCl}<2$ & 32,217 & $18.73 \%$ & 879,610 & $56.82 \%$ & 88,195 & $25.64 \%$ & 823,632 & 59.86 \\
\hline $\mathrm{CCl} \geq 2$ & 139,787 & $81.27 \%$ & 668,427 & $43.18 \%$ & 255,824 & $74.36 \%$ & 552,390 & 40.14 \\
\hline \multicolumn{9}{|l|}{ Charlson comorbidities } \\
\hline Myocardial infarction & 20,594 & $11.97 \%$ & 32,411 & $2.09 \%$ & 29,040 & $8.44 \%$ & 23,965 & $1.74 \%$ \\
\hline Congestive heart failure & 36,022 & $20.94 \%$ & 84,121 & $5.43 \%$ & 55,756 & $16.21 \%$ & 64,387 & $4.68 \%$ \\
\hline Peripheral vascular disease & 21,069 & $12.25 \%$ & 68,687 & $4.44 \%$ & 34,510 & $10.03 \%$ & 55,246 & $4.01 \%$ \\
\hline Cardiovascular disease & 31,358 & $18.23 \%$ & 109,220 & $7.06 \%$ & 53,770 & $15.63 \%$ & 86,808 & $6.31 \%$ \\
\hline
\end{tabular}


Table 1 Characteristics of the study sample, by cohort (Continued)

\begin{tabular}{|c|c|c|c|c|c|c|c|c|}
\hline Dementia & 2,419 & $1.41 \%$ & 11,390 & $0.74 \%$ & 4,765 & $1.39 \%$ & 9,044 & $0.66 \%$ \\
\hline Chronic pulmonary disease & 49,776 & $28.94 \%$ & 213,749 & $13.81 \%$ & 89,955 & $26.15 \%$ & 173,570 & $12.61 \%$ \\
\hline Rheumatological disease & 8,821 & $5.13 \%$ & 30,566 & $1.97 \%$ & 15,119 & $4.39 \%$ & 24,268 & $1.76 \%$ \\
\hline Peptic ulcer disease & 5,174 & $3.01 \%$ & 13,678 & $0.88 \%$ & 8,643 & $2.51 \%$ & 10,209 & $0.74 \%$ \\
\hline Mild liver disease & 3,654 & $2.12 \%$ & 6,305 & $0.41 \%$ & 5,380 & $1.56 \%$ & 4,579 & $0.33 \%$ \\
\hline Diabetes without chronic complications & 169,829 & $98.74 \%$ & $1,525,023$ & $98.51 \%$ & 339,677 & $98.74 \%$ & $1,355,175$ & $98.48 \%$ \\
\hline Diabetes with chronic complications & 45,140 & $26.24 \%$ & 219,433 & $14.17 \%$ & 83,992 & $24.41 \%$ & 180,581 & $13.12 \%$ \\
\hline Paraplegia & 3,765 & $2.19 \%$ & 4,538 & $0.29 \%$ & 5,139 & $1.49 \%$ & 3,164 & $0.23 \%$ \\
\hline Renal impairment & 17,789 & $10.34 \%$ & 29,344 & $1.90 \%$ & 26,180 & $7.61 \%$ & 20,953 & $1.52 \%$ \\
\hline Cancer & 34,466 & $20.04 \%$ & 101,118 & $6.53 \%$ & 54,085 & $15.72 \%$ & 81,499 & $5.92 \%$ \\
\hline Severe liver disease & 39,130 & $22.75 \%$ & 113,367 & $7.32 \%$ & 66,117 & $19.22 \%$ & 86,380 & $6.28 \%$ \\
\hline Metastatic cancer & 9,830 & $5.71 \%$ & 7,726 & $0.50 \%$ & 12,346 & $3.59 \%$ & 5,210 & $0.38 \%$ \\
\hline HIV/AIDS & 1,094 & $0.64 \%$ & 2,050 & $0.13 \%$ & 1,693 & $0.49 \%$ & 1,451 & $0.11 \%$ \\
\hline \multicolumn{9}{|l|}{ Antidiabetic agents received on Index } \\
\hline Glucagon-like peptide-1 & 991 & $0.58 \%$ & 7,500 & $0.48 \%$ & 2,390 & $0.69 \%$ & 6,101 & $0.44 \%$ \\
\hline Dipeptidyl peptidase-4 & 629 & $0.37 \%$ & 6,071 & $0.39 \%$ & 1,291 & $0.38 \%$ & 5,409 & $0.39 \%$ \\
\hline Biguanides & 21,285 & $12.37 \%$ & 218,752 & $14.13 \%$ & 46,060 & $13.39 \%$ & 193,977 & $14.10 \%$ \\
\hline Sulfonylureas & 14,169 & $8.24 \%$ & 122,570 & $7.92 \%$ & 29,290 & $8.51 \%$ & 107,449 & $7.81 \%$ \\
\hline Thiazolidinedione & 9,976 & $5.80 \%$ & 85,809 & $5.54 \%$ & 23,079 & $6.71 \%$ & 72,706 & $5.28 \%$ \\
\hline Meglitinides & 1,045 & $0.61 \%$ & 7,266 & $0.47 \%$ & 2,491 & $0.72 \%$ & 5,820 & $0.42 \%$ \\
\hline Alpha-glucosidase inhibitors & 187 & $0.11 \%$ & 1,435 & $0.09 \%$ & 460 & $0.13 \%$ & 1,162 & $0.08 \%$ \\
\hline Insulin & 14,220 & $8.27 \%$ & 70,542 & $4.56 \%$ & 29,921 & $8.70 \%$ & 54,841 & $3.99 \%$ \\
\hline Other antidiabetic agents & 8,689 & $5.05 \%$ & 79,749 & $5.15 \%$ & 19,045 & $5.54 \%$ & 69,393 & $5.04 \%$ \\
\hline
\end{tabular}

$\mathrm{CCl}=$ Charlson Comorbidity Index; $\mathrm{SD}=$ standard deviation .

${ }^{a} \mathrm{CCl}$ score calculated in the 1-year post-index date period.

distribution was approximately equal. The mean (SD) CCI score was greater among patients in the top $20 \%$ of the cost distribution (3.7 [2.8]), than among patients in the bottom $80 \%$ of the cost distribution (2.0 [1.6]). Chronic pulmonary disease, liver disease, and congestive heart failure were the most common conditions in both cohorts. The percentage of patients receiving oral antidiabetic medications at index date was approximately the same in both cohorts; however, more than twice the percentage of patients in the top $20 \%$ of the cost distribution received insulin, compared with patients in the bottom $80 \%$ of the cost distribution (8.7\% vs. $4.0 \%)$.

Patients in the top $10 \%$ of the cost distribution were found to be similar to patients in the top $20 \%$ of the cost distribution in terms of mean age, sex distribution, geographic region, health plan type, and payer type. The mean (SD) CCI score was greater for patients in the top $10 \%$ of the cost distribution (4.3 [3.0]), with a greater percentage of these patients having nearly all of the individual CCI components, than for patients in the top $20 \%$ of the cost distribution (2.1 [1.7]). Additionally, approximately the same percentage of patients in the top $10 \%$ of the cost distribution received oral antidiabetic medications and insulin as in the top $20 \%$ of the cost distribution.

The strongest predictor of being an HC patient (either in the top $20 \%$ or top $10 \%$ of the cost distribution) was having a CCI score $\geq 2$ (odds ratio [OR] for top 20\% regression vs. top $10 \%$ regression: 3.9 vs. 4.9 ; both $P<0.0001$ ) (Table 2). Additionally, a diagnosis of renal impairment (OR for top $20 \%$ regression vs. top $10 \%$ regression: 2.2 vs. 2.4 ; both $P<0.0001$ ), a diagnosis of obesity (OR for top $20 \%$ regression vs. top $10 \%$ regression: 2.0 vs. 2.1 ; both $P<0.0001$ ), receipt of insulin (OR for top 20\% regression vs. top $10 \%$ regression: 2.7 vs. 2.1 ; both $P<0.0001)$, and a diagnosis of hypertension (OR for top $20 \%$ regression vs. top $10 \%$ regression: 1.5 vs. 1.6 ; both $P<0.0001$ ) were all found to be associated with a significant increase in the odds of being an $\mathrm{HC}$ patient.

Patients in the top $20 \%$ of the cost distribution had total all-cause costs that were, on average, $\$ 32,179$ more than the costs accrued by patients in the bottom $80 \%$ of the cost distribution (mean [SD] all-cause costs, top 20\% vs. bottom $80 \%$ : $\$ 35,596[\$ 50,903]$ vs. $\$ 3,417[\$ 2,775]$ ) (Table 3). Furthermore, patients in the top $10 \%$ of the 
Table 2 Predictors of being an HC T2DM patient, among all patients with T2DM ${ }^{a}$

\begin{tabular}{|c|c|c|c|c|c|c|c|c|}
\hline \multirow[t]{3}{*}{ Variable } & \multicolumn{8}{|l|}{ Cohort } \\
\hline & \multicolumn{4}{|c|}{ Top $10 \%$ of all-cause costs } & \multicolumn{4}{|c|}{ Top $20 \%$ of all-cause costs } \\
\hline & Odds ratio & Lower $95 \% \mathrm{Cl}$ & Upper $95 \% \mathrm{Cl}$ & $P$ value & Odds ratio & Lower $95 \% \mathrm{Cl}$ & Upper $95 \% \mathrm{Cl}$ & $P$ value \\
\hline \multicolumn{9}{|l|}{ Age, in years (vs. $55+$ years) } \\
\hline$<35$ & 1.128 & 1.098 & 1.159 & $<0.0001$ & 1.311 & 1.286 & 1.337 & $<0.0001$ \\
\hline $35-44$ & 1.063 & 1.043 & 1.085 & $<0.0001$ & 1.094 & 1.078 & 1.110 & $<0.0001$ \\
\hline $45-54$ & 1.200 & 1.184 & 1.216 & $<0.0001$ & 1.179 & 1.167 & 1.191 & $<0.0001$ \\
\hline Female (vs. male) & 1.023 & 1.012 & 1.034 & $<0.0001$ & 1.175 & 1.165 & 1.184 & $<0.0001$ \\
\hline \multicolumn{9}{|l|}{ Geographic region (vs. East) } \\
\hline South & 0.814 & 0.800 & 0.827 & $<0.0001$ & 0.763 & 0.754 & 0.773 & $<0.0001$ \\
\hline Midwest & 1.166 & 1.150 & 1.183 & $<0.0001$ & 1.156 & 1.144 & 1.168 & $<0.0001$ \\
\hline West & 1.107 & 1.088 & 1.128 & $<0.0001$ & 1.047 & 1.033 & 1.062 & $<0.0001$ \\
\hline \multicolumn{9}{|l|}{$\begin{array}{l}\text { Health plan type (vs. health } \\
\text { maintenance organization) }\end{array}$} \\
\hline Preferred provider organization & 1.091 & 1.074 & 1.108 & $<0.0001$ & 1.041 & 1.029 & 1.054 & $<0.0001$ \\
\hline Point of service & 1.141 & 1.119 & 1.163 & $<0.0001$ & 1.080 & 1.063 & 1.096 & $<0.0001$ \\
\hline Indemnity & 0.510 & 0.499 & 0.521 & $<0.0001$ & 0.608 & 0.598 & 0.618 & $<0.0001$ \\
\hline \multicolumn{9}{|l|}{ Payer type (vs. commercial) } \\
\hline Medicaid & 1.221 & 1.170 & 1.274 & $<0.0001$ & 1.198 & 1.157 & 1.239 & $<0.0001$ \\
\hline Medicare or Medicare Gap & 1.070 & 1.050 & 1.089 & $<0.0001$ & 0.978 & 0.964 & 0.992 & 0.0024 \\
\hline Self & 0.885 & 0.868 & 0.902 & $<0.0001$ & 0.967 & 0.953 & 0.981 & $<0.0001$ \\
\hline $\begin{array}{l}\text { Charlson Comorbidity Index } \\
\text { score } \geq 2 \text { (vs. < 2) }\end{array}$ & 4.896 & 4.832 & 4.961 & $<0.0001$ & 3.908 & 3.873 & 3.944 & $<0.0001$ \\
\hline $\begin{array}{l}\text { Renal impairment diagnosis } \\
\text { (vs. no renal impairment diagnosis) }\end{array}$ & 2.368 & 2.333 & 2.404 & $<0.0001$ & 2.179 & 2.150 & 2.208 & $<0.0001$ \\
\hline $\begin{array}{l}\text { Hypertension diagnosis } \\
\text { (vs. no hypertension diagnosis) }\end{array}$ & 1.625 & 1.602 & 1.648 & $<0.0001$ & 1.519 & 1.504 & 1.535 & $<0.0001$ \\
\hline $\begin{array}{l}\text { Obesity diagnosis } \\
\text { (vs. no obesity diagnosis) }\end{array}$ & 2.106 & 2.076 & 2.13741 & $<0.0001$ & 2.033 & 2.009 & 2.056 & $<0.0001$ \\
\hline \multicolumn{9}{|l|}{$\begin{array}{l}\text { Antidiabetic treatment } \\
\text { (vs. no treatment) }\end{array}$} \\
\hline Received insulin & 2.098 & 2.068 & 2.128 & $<0.0001$ & 2.744 & 2.7142 & 2.775 & $<0.0001$ \\
\hline $\begin{array}{l}\text { Received oral antidiabetic } \\
\text { agents only }\end{array}$ & 1.110 & 1.097 & 1.124 & $<0.0001$ & 1.283 & 1.271 & 1.294 & $<0.0001$ \\
\hline
\end{tabular}

$\mathrm{Cl}=$ confidence interval; $\mathrm{HC}=$ high cost; $\mathrm{T} 2 \mathrm{DM}=$ type 2 diabetes mellitus.

${ }^{a} 28,100$ patients were excluded from the regression analysis because they were missing age, sex, health plan, or payer information.

cost distribution had total all-cause costs that were, on average, $\$ 51,794$ more than the costs accrued by patients in the bottom $90 \%$ of the cost distribution (i.e., mean [SD] all-cause costs, top $10 \%$ vs. bottom $90 \%$ : $\$ 56,468$ $[\$ 65,604]$ vs. $\$ 4,674$ [ $\$ 4,504]$. Additionally, all-cause costs for patients in the top $10 \%$ of the cost distribution were approximately $\$ 20,872$ more than all-cause costs for patients in the top $20 \%$ of the cost distribution.

All-cause inpatient visits were responsible for approximately $42 \%$ of the difference in all-cause costs between patients in the top $20 \%$ and patients in the bottom $80 \%$ of the cost distribution, with $55.8 \%$ of patients in the top $20 \%$ of the cost distribution having at least one all-cause hospitalization and $21.0 \%$ of patients having two or more hospitalizations compared with $7.4 \%$ of patients in the bottom $80 \%$ of the cost distribution having at least one all-cause hospitalization and $1.3 \%$ of patients having two or more hospitalizations. Similarly, all-cause inpatient visits were responsible for approximately $47 \%$ of the difference in costs between patients in the top 10\% and patients in the bottom $90 \%$ of the distribution, with $74.2 \%$ of patients in the top $10 \%$ of the distribution having at least one all-cause hospitalization and $34.4 \%$ of patients having two or more hospitalizations, compared with only $10.8 \%$ of patients in the bottom $90 \%$ of the distribution having at 
Table 3 Summary of overall health care utilization and costs during the 12-month follow-up period, by cohort

\begin{tabular}{|c|c|c|c|c|c|c|c|c|}
\hline \multirow{2}{*}{$\overline{\text { Inpatient services }}$} & \multicolumn{8}{|l|}{ Cohort } \\
\hline & \multicolumn{2}{|c|}{ Top $10 \%$ of costs } & \multicolumn{2}{|c|}{ Bottom $90 \%$ of costs } & \multicolumn{2}{|c|}{ Top $20 \%$ of costs } & \multicolumn{2}{|c|}{ Bottom $80 \%$ of costs } \\
\hline Had $\geq 1$ hospital admission (n, \%) & 127,553 & $74.16 \%$ & 166,729 & $10.77 \%$ & 192,070 & $55.83 \%$ & 102,212 & $7.43 \%$ \\
\hline Had $\geq 2$ hospital admissions ( $n, \%)$ & 59,199 & $34.42 \%$ & 31,289 & $2.02 \%$ & 72,263 & $21.01 \%$ & 18,225 & $1.32 \%$ \\
\hline Mean number of admissions (SD) & 1.44 & $(1.62)$ & 0.14 & $(0.45)$ & 0.97 & $(1.36)$ & 0.09 & $(0.37)$ \\
\hline Mean (SD) inpatient days ${ }^{\mathrm{a}}$ & 13.81 & $(24.88)$ & 7.07 & $(39.57)$ & 11.65 & $(22.04)$ & 6.89 & $(49.31)$ \\
\hline Mean (SD) total costs & $\$ 24,766$ & $(\$ 48,149)$ & $\$ 427$ & $(\$ 1,739)$ & $\$ 13,618$ & $(\$ 35,945)$ & $\$ 171$ & $(\$ 805)$ \\
\hline \multicolumn{9}{|l|}{ SNF stays } \\
\hline Had $\geq 1$ SNF admission (n, \%) & 10,410 & $6.05 \%$ & 8,987 & $0.58 \%$ & 14,047 & $4.08 \%$ & 5,350 & $0.39 \%$ \\
\hline Mean number of SNF admissions (SD) & 0.10 & $(0.49)$ & 0.01 & $(0.14)$ & 0.07 & $(0.41)$ & 0.01 & $(0.10)$ \\
\hline Mean (SD) SNF days ${ }^{a}$ & 33.70 & $(44.70)$ & 48.52 & (203.18) & 42.75 & $(135.81)$ & 34.84 & $(157.98)$ \\
\hline Mean (SD) total costs & $\$ 525$ & $(\$ 3,532)$ & $\$ 20$ & $(\$ 389)$ & $\$ 323$ & $(\$ 2,612)$ & $\$ 7$ & $(\$ 180)$ \\
\hline \multicolumn{9}{|l|}{ ED visits } \\
\hline $\operatorname{Had} \geq 1$ ED visit $(n, \%)$ & 103,348 & $60.08 \%$ & 355,475 & $22.96 \%$ & 183,931 & $53.47 \%$ & 274,892 & $19.98 \%$ \\
\hline Mean number of ED visits (SD) & 5.31 & $(14.32)$ & 1.29 & $(4.60)$ & 4.26 & $(11.65)$ & 1.05 & $(3.90)$ \\
\hline Mean (SD) total costs & $\$ 888$ & $(\$ 2,283)$ & $\$ 136$ & $(\$ 505)$ & $\$ 659$ & $(\$ 1,791)$ & $\$ 99$ & $(\$ 370)$ \\
\hline \multicolumn{9}{|l|}{ Office visits } \\
\hline Had $\geq 1$ office visit (n, \%) & 168,403 & $97.91 \%$ & $1,505,071$ & $97.22 \%$ & 336,935 & $97.94 \%$ & $1,336,539$ & $97.13 \%$ \\
\hline Mean number of office visits (SD) & 26.00 & $(20.97)$ & 12.72 & $(12.11)$ & 23.73 & $(19.25)$ & 11.62 & $(10.86)$ \\
\hline Mean (SD) total costs & $\$ 5,827$ & $(\$ 13,092)$ & $\$ 1,155$ & $(\$ 1,440)$ & $\$ 4,246$ & $(\$ 9,562)$ & $\$ 967$ & $(\$ 1,098)$ \\
\hline \multicolumn{9}{|l|}{ Pharmacy } \\
\hline Had $\geq 1$ prescription $(n, \%)$ & 150,474 & $87.48 \%$ & $1,225,785$ & $79.18 \%$ & 302,929 & $88.06 \%$ & $1,073,330$ & $78.00 \%$ \\
\hline Mean number of prescriptions (SD) & 53.72 & $(44.11)$ & 27.99 & $(28.83)$ & 50.37 & $(41.21)$ & 25.61 & $(26.56)$ \\
\hline Mean (SD) total costs & $\$ 4,854$ & $(\$ 12,486)$ & $\$ 1,428$ & $(\$ 2,162)$ & $\$ 4,190$ & $(\$ 9,270)$ & $\$ 1,166$ & $(\$ 1,660)$ \\
\hline \multicolumn{9}{|l|}{ Outpatient hospital visits } \\
\hline Had $\geq 1$ outpatient visit (n, \%) & 131,885 & $76.68 \%$ & 844,910 & $54.58 \%$ & 257,619 & $74.89 \%$ & 719,176 & $52.26 \%$ \\
\hline Mean number of outpatient visits (SD) & 43.33 & $(82.58)$ & 9.15 & $(20.37)$ & 32.64 & $(65.27)$ & 7.55 & $(16.13)$ \\
\hline Mean (SD) total costs & $\$ 8,763$ & $(\$ 22,823)$ & $\$ 767$ & $(\$ 1,816)$ & $\$ 5,807$ & $(\$ 16,625)$ & $\$ 507$ & $(\$ 1,136)$ \\
\hline \multicolumn{9}{|l|}{ Laboratory services } \\
\hline Had $\geq 1$ laboratory service $(n, \%)$ & 92,529 & $53.79 \%$ & 655,781 & $42.36 \%$ & 178,840 & $51.99 \%$ & 569,470 & $41.39 \%$ \\
\hline Mean number of laboratory services (SD) & 13.13 & $(30.85)$ & 5.50 & $(11.32)$ & 10.72 & $(24.64)$ & 5.15 & $(10.57)$ \\
\hline Mean (SD) total costs & $\$ 247$ & $(\$ 810)$ & $\$ 75$ & $(\$ 210)$ & $\$ 194$ & $(\$ 630)$ & $\$ 66$ & $(\$ 179)$ \\
\hline \multicolumn{9}{|l|}{ OOP services } \\
\hline Had $\geq 1$ OOP services $(n, \%)$ & 153,936 & $89.50 \%$ & 834,883 & $53.93 \%$ & 293,499 & $85.31 \%$ & 695,320 & $50.53 \%$ \\
\hline Mean number of OOP services (SD) & 46.30 & $(95.37)$ & 8.49 & $(22.41)$ & 34.05 & $(75.59)$ & 6.82 & $(16.91)$ \\
\hline Mean (SD) total costs & $\$ 10,598$ & $(\$ 29,858)$ & $\$ 666$ & $(\$ 1,633)$ & $\$ 6,559$ & $(\$ 21,633)$ & $\$ 434$ & $(\$ 1,016)$ \\
\hline \multicolumn{9}{|l|}{ Total health care utilization } \\
\hline Had $\geq 1$ medical encounter $(n, \%)$ & 172,004 & $100.00 \%$ & $1,548,037$ & $100.00 \%$ & 344,019 & $100.00 \%$ & $1,376,022$ & $100.00 \%$ \\
\hline Mean number of encounters (SD) & 189.33 & $(150.55)$ & 65.29 & $(51.82)$ & 156.81 & $(123.32)$ & 57.92 & $(42.74)$ \\
\hline Mean (SD) total costs & $\$ 56,468$ & $(\$ 65,604)$ & $\$ 4,674$ & $(\$ 4,504)$ & $\$ 35,596$ & $(\$ 50,903)$ & $\$ 3,417$ & $(\$ 2,775)$ \\
\hline
\end{tabular}

$\mathrm{ED}=$ emergency department; $\mathrm{OOP}=$ other outpatient; $\mathrm{SD}=$ standard deviation; $\mathrm{SNF}=$ skilled nursing facility .

${ }^{a}$ Mean inpatient and SNF days estimated among only those patients with at least 1 unique admission. 
least one all-cause hospitalization and $2.0 \%$ of patients having two or more hospitalizations.

All-cause outpatient hospital visits contributed to approximately $16.5 \%$ of the difference in costs between patients in the top $20 \%$ and patients in the bottom $80 \%$ of the cost distribution, with $74.9 \%$ of patients in the top $20 \%$ of the cost distribution having at least one all-cause outpatient hospital visit compared with $52.3 \%$ of patients in the bottom $80 \%$ of the cost distribution. Outpatient hospital visits had a similar contribution to the difference in costs between patients in the top 10\% and patients in the bottom $90 \%$ of the cost distribution. Specifically, all-cause outpatient hospital visits contributed to approximately $15.4 \%$ of the difference in all-cause costs between patients in the top $10 \%$ of the cost distribution and patients in the bottom $90 \%$ of the cost distribution, with $76.7 \%$ of patients in the top $10 \%$ of the cost distribution having at least one all-cause outpatient hospital visit, compared with $54.6 \%$ of patients in the bottom $90 \%$ of the all-cause cost distribution.

All-cause prescription fills and all-cause office visits contributed to $9.4 \%$ and $10.2 \%$ of the difference in allcause costs among patients in the top $20 \%$ and bottom $80 \%$, respectively, of the cost distribution. All-cause prescription fills and all-cause office visits contributed to $6.6 \%$ and $9.0 \%$ of the difference among patients in the top $10 \%$ and bottom $90 \%$, respectively, of the cost distribution. However, patients in both the top $20 \%$ and top $10 \%$ of the cost distribution filled approximately twice as many prescriptions and had almost double the number of physician office visits, compared with patients in the bottom $80 \%$ and bottom $90 \%$ of the cost distribution (top $20 \%$ vs. bottom $80 \%$ : 50.4 vs. 25.6 prescription fills; 23.7 vs. 11.6 office visits: top $10 \%$ vs. bottom $90 \%$ : 53.7 vs. 28.0 prescription fills; 26.0 vs. 12.7 office visits).

Health care costs related to T2DM were, on average, $\$ 2,977$ more for patients in the top $20 \%$ of the cost distribution than for patients in the bottom $80 \%$ of the cost distribution and \$4,136 more for patients in the top 10\% of the cost distribution than for patients in the bottom $90 \%$ of the cost distribution (Table 4). Mean (SD) T2DMrelated costs among patients in the top $20 \%$ of the cost distribution were $\$ 3,780(\$ 8,530)$, which represented $10.6 \%$ of all-cause costs; T2DM-related costs among patients in the bottom $80 \%$ of the cost distribution were $\$ 803(\$ 1,065)$, which represented $23.5 \%$ of all-cause costs. Similarly, mean (SD) T2DM-related costs among patients in the top $10 \%$ of the cost distribution were $\$ 5,121$ ( $\$ 11,575)$, which represented $9.1 \%$ of all-cause costs; T2DM-related costs among patients in the bottom $90 \%$ of the cost distribution were $\$ 985(\$ 1,469)$, which represented $21.1 \%$ of all-cause costs. Unlike all-cause costs, the biggest difference in T2DMrelated costs between patients in the top $20 \%$ and $10 \%$ and patients in bottom $80 \%$ and $90 \%$ of the cost distribution was outpatient hospital visits, which accounted for approximately $25 \%$ of the cost difference in both groups.

The entire T2DM population included in this study $(\mathrm{N}=1,720,041)$ accrued all-cause costs of approximately $\$ 17$ billion (Figures 1 and 2). The top 10\% of patients accrued costs of more than $\$ 9.7$ billion, which represented more than $57 \%$ of the costs accrued by this population. The top 20\% of patients accrued costs of more than $\$ 12$ billion, which represented more than $72 \%$ of the costs accrued by this population. In the overall population of patients, over $\$ 2.4$ billion of the total all-cause costs could be directly linked to T2DM (i.e., $14.2 \%$ of all-cause costs accrued by this population were attributable directly to T2DM). The top $10 \%$ of patients accrued T2DM-related costs of $\$ 880$ million, which represented $36.6 \%$ of the total T2DM-related costs, while the top $20 \%$ of patients accrued T2DM-related costs of $\$ 1.3$ billion, which represented $54.1 \%$ of the total T2DM-related costs.

\section{Discussion}

This study examined patients with T2DM in a large, managed care population and quantified differences in health care costs by categories of cost distributions. Patients were identified as being $\mathrm{HC}$ if their total care costs fell in the top $10 \%$ or the top $20 \%$ of the total cost distribution. Patients in the top $10 \%$ of the total cost distribution accrued annual per-patient health care costs that were on average $\$ 50,000$ more than the annual perpatient health care costs accrued by patients in the bottom $90 \%$ of the total cost distribution. Similarly, patients in the top $20 \%$ of the total cost distribution accrued annual per-patient health care costs that were over $\$ 32,000$ more than the annual per-patient health care costs accrued by patients in the bottom $80 \%$ of the total cost distribution. Multivariable logistic regression models found that patients were significantly more likely to be in the top $10 \%$ or the top $20 \%$ of the total cost distribution if they had a CCI score $\geq 2$; had received a diagnosis of renal impairment, obesity, or hypertension; or were treated with insulin.

Data drawn from the Medical Expenditure Panel Survey have previously shown that a small percentage of patients typically account for a large percentage of health care costs. Specifically, using data from 1999, the survey found that, in the general community population, more than half of the total health care spending was accrued by only $5 \%$ of the population [16]. The Olin study supports the rule of thumb that $20 \%$ of patients consume $80 \%$ of health care resources. Additionally, Conwell and Cohen, using data from a 2002 US noninstitutionalized population, found that exactly $20 \%$ of patients accrued $80 \%$ of costs [14]. Similarly, we found that patients in the top $20 \%$ of the total cost distribution accrued costs of $\$ 12.2$ billion annually, which represented $72 \%$ of the 
Table 4 Summary of diabetes-related health care utilization and costs during the 12-month follow-up period, by cohort

\begin{tabular}{|c|c|c|c|c|c|c|c|c|}
\hline \multirow{2}{*}{ Diabetes-related inpatient stays } & \multicolumn{8}{|l|}{ Cohort } \\
\hline & \multicolumn{2}{|c|}{ Top $10 \%$ of costs } & \multicolumn{2}{|c|}{ Bottom $90 \%$ of costs } & \multicolumn{2}{|c|}{ Top $20 \%$ of costs } & \multicolumn{2}{|c|}{ Bottom $80 \%$ of costs } \\
\hline Had $\geq 1$ hospital admission ( $n, \%)$ & 30,310 & $17.62 \%$ & 27,729 & $1.79 \%$ & 41,220 & $11.98 \%$ & 16,819 & $1.22 \%$ \\
\hline Mean number of hospital admissions (SD) & 0.23 & $(0.60)$ & 0.02 & $(0.15)$ & 0.15 & $(0.48)$ & 0.01 & $(0.12)$ \\
\hline Mean (SD) inpatient days ${ }^{\mathrm{a}}$ & 6.93 & $(10.48)$ & 4.29 & $(7.60)$ & 6.28 & $(9.68)$ & 4.17 & (8.16) \\
\hline Mean costs (SD) & $\$ 677$ & $(\$ 5,515)$ & $\$ 20$ & $(\$ 331)$ & $\$ 387$ & $(\$ 3,953)$ & $\$ 10$ & $(\$ 194)$ \\
\hline \multicolumn{9}{|l|}{ Diabetes-related SNF stays ( $n, \%)$} \\
\hline Had $\geq 1$ SNF admission & 1,802 & $1.05 \%$ & 1,405 & $0.09 \%$ & 2,468 & $0.72 \%$ & 739 & $0.05 \%$ \\
\hline Mean number of SNF admissions (SD) & 0.01 & $(0.14)$ & 0.00 & $(0.04)$ & 0.01 & $(0.12)$ & 0.00 & $(0.03)$ \\
\hline Mean (SD) SNF days ${ }^{\mathrm{a}}$ & 12.96 & (31.56) & 22.29 & $(139.07)$ & 17.53 & $(105.76)$ & 15.41 & $(43.85)$ \\
\hline Mean costs (SD) & $\$ 14$ & $(\$ 424)$ & $\$ 1$ & $(\$ 76)$ & $\$ 9$ & $(\$ 332)$ & $\$ 0$ & $(\$ 37)$ \\
\hline \multicolumn{9}{|l|}{ Diabetes-related ED visits } \\
\hline $\operatorname{Had} \geq 1$ ED visit $(n, \%)$ & 39,813 & $23.15 \%$ & 132,909 & $8.59 \%$ & 70,699 & $20.55 \%$ & 102,023 & $7.41 \%$ \\
\hline Mean number of ED visits (SD) & 1.36 & $(5.88)$ & 0.38 & $(2.37)$ & 1.14 & $(5.03)$ & 0.31 & $(2.07)$ \\
\hline Mean costs (SD) & $\$ 232$ & $(\$ 1,003)$ & $\$ 42$ & $(\$ 277)$ & $\$ 182$ & $(\$ 825)$ & $\$ 31$ & $(\$ 204)$ \\
\hline \multicolumn{9}{|l|}{ Diabetes-related office visits } \\
\hline Had $\geq 1$ office visit (n, \%) & 137,104 & $79.71 \%$ & $1,273,378$ & $82.26 \%$ & 277,094 & $80.55 \%$ & $1,133,388$ & $82.37 \%$ \\
\hline Mean number of office visits (SD) & 4.28 & $(4.59)$ & 3.42 & $(3.32)$ & 4.23 & $(4.39)$ & 3.33 & (3.18) \\
\hline Mean costs (SD) & $\$ 469$ & $(\$ 1,290)$ & $\$ 245$ & $(\$ 357)$ & $\$ 424$ & $(\$ 1,002)$ & $\$ 228$ & $(\$ 314)$ \\
\hline \multicolumn{9}{|l|}{ Diabetes-related pharmacy } \\
\hline Had $\geq 1$ prescription $(n, \%)$ & 107,729 & $62.63 \%$ & 818,451 & $52.87 \%$ & 219,248 & $63.73 \%$ & 706,932 & $51.38 \%$ \\
\hline Mean number of prescriptions (SD) & 6.98 & $(8.57)$ & 5.49 & $(7.72)$ & 7.29 & $(8.78)$ & 5.22 & $(7.51)$ \\
\hline Mean costs (SD) & $\$ 636$ & $(\$ 1,660)$ & $\$ 356$ & $(\$ 806)$ & $\$ 702$ & $(\$ 1,511)$ & $\$ 304$ & $(\$ 694)$ \\
\hline \multicolumn{9}{|l|}{ Diabetes-related outpatient hospital visits } \\
\hline Had $\geq 1$ outpatient visit (n, \%) & 69,070 & $40.16 \%$ & 411,072 & $26.55 \%$ & 131,277 & $38.16 \%$ & 348,865 & $25.35 \%$ \\
\hline Mean number of outpatient visits (SD) & 6.18 & $(18.89)$ & 2.46 & $(7.63)$ & 5.23 & $(16.47)$ & 2.23 & $(6.49)$ \\
\hline Mean (SD) total costs & $\$ 1,204$ & $(\$ 5,433)$ & $\$ 145$ & $(\$ 652)$ & $\$ 849$ & $(\$ 4,007)$ & $\$ 101$ & $(\$ 408)$ \\
\hline \multicolumn{9}{|l|}{ Diabetes-related laboratory services } \\
\hline Had $\geq 1$ laboratory service (n, \%) & 51,096 & $29.71 \%$ & 439,858 & $28.41 \%$ & 101,715 & $29.57 \%$ & 389,239 & $28.29 \%$ \\
\hline Mean number of laboratory services (SD) & 3.52 & $(9.92)$ & 2.76 & $(6.48)$ & 3.35 & $(8.82)$ & 2.71 & $(6.32)$ \\
\hline Mean (SD) total costs & $\$ 48$ & $(\$ 204)$ & $\$ 29$ & $(\$ 90)$ & $\$ 43$ & $(\$ 169)$ & $\$ 28$ & $(\$ 85)$ \\
\hline \multicolumn{9}{|l|}{ Diabetes-related OOP services } \\
\hline Had $\geq 1$ OOP service $(n, \%)$ & 80,576 & $46.85 \%$ & 414,844 & $26.80 \%$ & 147,877 & $42.99 \%$ & 347,543 & $25.26 \%$ \\
\hline Mean number of OOP services (SD) & 8.11 & $(24.22)$ & 2.29 & $(7.32)$ & 6.33 & $(19.66)$ & 2.01 & $(6.09)$ \\
\hline Mean costs (SD) & $\$ 1,842$ & $(\$ 7,754)$ & $\$ 147$ & $(\$ 659)$ & $\$ 1,182$ & $(\$ 5,634)$ & $\$ 100$ & $(\$ 398)$ \\
\hline \multicolumn{9}{|l|}{ Diabetes-related total health care utilization } \\
\hline Had $\geq 1$ medical encounter ( $n, \%)$ & 168,326 & $97.86 \%$ & $1,541,265$ & $99.56 \%$ & 338,383 & $98.36 \%$ & $1,371,208$ & $99.65 \%$ \\
\hline Mean number of encounters (SD) & 30.68 & $(37.16)$ & 16.83 & $(16.26)$ & 27.72 & $(31.67)$ & 15.84 & $(14.58)$ \\
\hline Mean costs (SD) & $\$ 5,121$ & $(\$ 11,575)$ & $\$ 985$ & $(\$ 1,469)$ & $\$ 3,780$ & $(\$ 8,530)$ & $\$ 803$ & $(\$ 1,065)$ \\
\hline
\end{tabular}

$\mathrm{ED}=$ emergency department; $\mathrm{OOP}=$ other outpatient; $\mathrm{SD}=$ standard deviation; $\mathrm{SNF}=$ skilled nursing facility. ${ }^{a}$ Mean inpatient and SNF days estimated among only patients with at least 1 unique admission. 


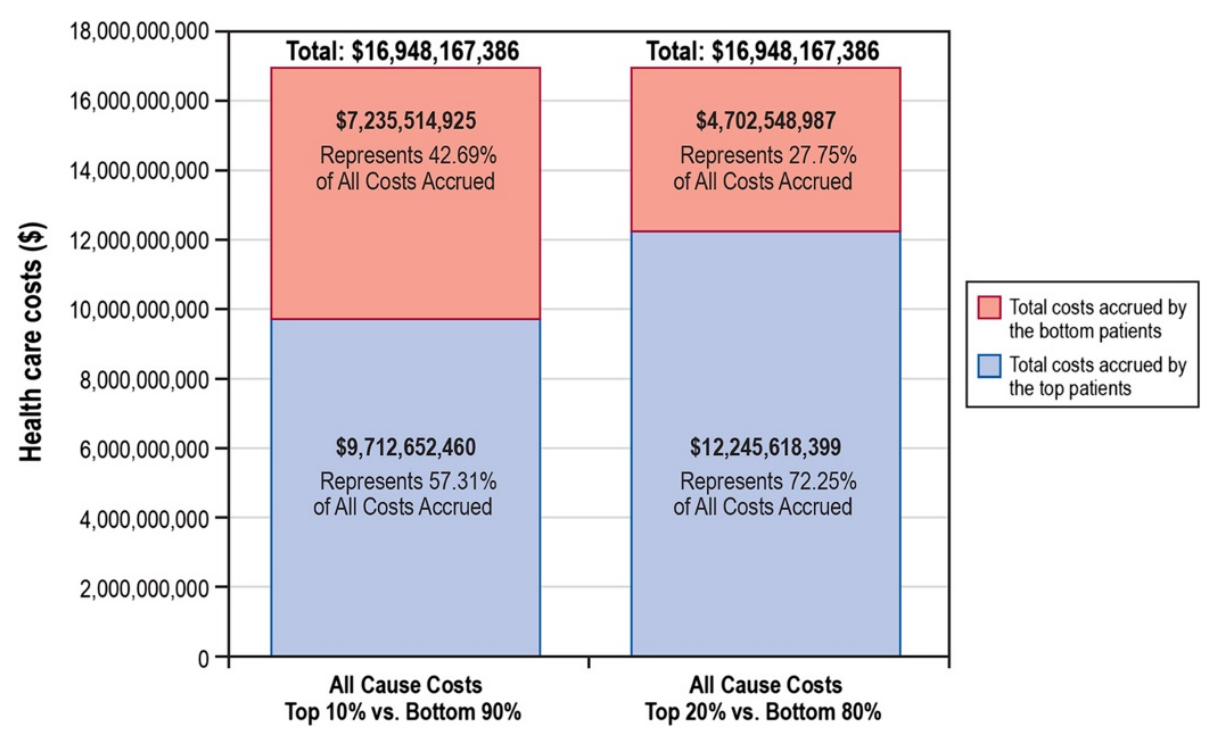

Figure 1 Descriptive summary of all-cause health care costs during the 12-month follow-up period.

total costs accrued by the T2DM population. Additionally, we found that patients in the top $10 \%$ of the total cost distribution accrued costs of $\$ 9.7$ billion annually, which represented $57 \%$ of the total costs accrued by the T2DM population.

This study used methodology similar to the approach described by Etemad and McCollam in an article examining predictors of $\mathrm{HC}$ managed care patients with acute coronary syndrome [13]. Etemad and McCollam identified patients with newly onset acute coronary syndrome and assessed these patients' health care costs over 12 months after disease onset. The authors classified patients as being
$\mathrm{HC}$ if the patients accrued costs in the top $20 \%$ of the population; multivariable regression analyses were estimated to assess predictors of being an $\mathrm{HC}$ patient. Similar to our study, many of the factors associated with being an $\mathrm{HC}$ patient in the Etemad analysis were nonmodifiable comorbidities such as hypertension, diabetes, or pulmonary disease.

Etemad and McCollam also observed that an initial hospitalization for acute coronary syndrome had costs that were equal to nearly two-thirds of the costs accrued in the entire year following hospital discharge. Although our study used a slightly different methodology (we

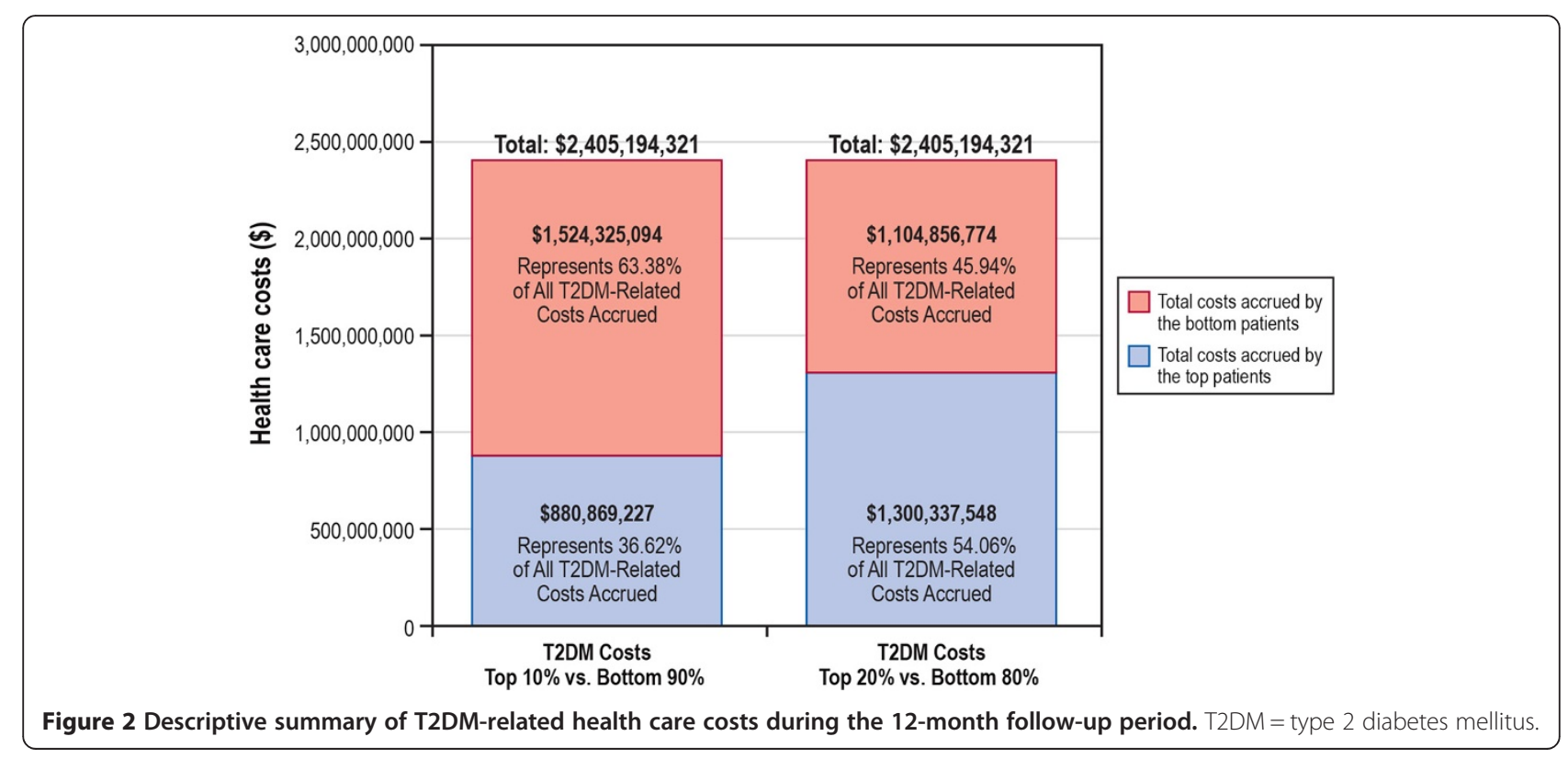


examined all inpatient stays vs. a single initial inpatient stay) to that of Etemad and McCollam, we found that approximately $40 \%$ of the health care costs accrued in $\mathrm{HC}$ patients were associated with inpatient visits.

Hartmann [17] examined patients in the top decile of health care spending, using German health insurance information. Consistent with our analysis, Hartmann found that the highest health care expenses for patients were incurred in the inpatient sector, with over $80 \%$ of all $\mathrm{HC}$ patients having at least one hospital admission (compared with $74.2 \%$ in our analysis) [17]. Additionally, Hartmann [17] found that the reasons for the hospitalization differed based on patient age and sex, which further highlights the facts that $\mathrm{HC}$ patients require care tailored to their unique situation and that no single intervention exists that will reduce health care costs among all patients.

A previous study examining Medicare patients with T2DM found that interventions aimed at diabetes have not differed based on comorbid illness burden [18]. Our analysis found that patients with a higher comorbidity burden and more concomitant conditions were significantly more likely to be $\mathrm{HC}$. Therefore, from the perspective of a payer, one practical implication of the present analysis is that it may make sense to provide those patients who have the most comorbidities and concomitant conditions (i.e., those patients who are at the greatest risk of being $\mathrm{HC}$ ) with additional patient care tailored at treating the comorbidity or concomitant condition (e.g., weight loss programs for obese patients).

Sensitivity analyses were conducted, examining the 926,180 patients who received an antidiabetic medication (i.e., either an oral antidiabetic or insulin). In this subpopulation of treated T2DM patients, those with costs greater than $\$ 22,646$ comprised the top 10th percentile (vs. $\$ 20,528$ in the overall T2DM population), while patients with costs greater than $\$ 12,349$ comprised the top 20th percentile (vs. $\$ 10,901$ in the overall T2DM population). We found that there were no differences in patient demographics between the overall study sample and those patients who received antidiabetic medication. Predictors of being an HC T2DM patient were the same for the treated and overall T2DM populations. Specifically, in the treated T2DM population, having a CCI score greater than or equal to 2 was the strongest predictor of being an $\mathrm{HC}$ patient $(\mathrm{OR}=4.862 ; P<0.001)$, followed by a renal impairment diagnosis $(\mathrm{OR}=2.369 ; P<0.001)$, an obesity diagnosis $(\mathrm{OR}=1.991 ; P<0.001)$, or receipt of insulin $(\mathrm{OR}=1.897 ; P<0.001)$. Treated patients in the top $10 \%$ of the cost distribution accrued approximately $\$ 53,917$ more in health care costs versus treated patients in the bottom $90 \%$ of the cost distribution (vs. $\$ 51,794$ more in costs in the overall T2DM population), with the largest difference in costs attributable to inpatient stays. Additionally, treated patients in the top $10 \%$ of the cost distribution accrued costs of over $\$ 5.5$ billion, which represented $54.1 \%$ of all costs accrued by the treated T2DM population (vs. 57.3\% among all T2DM patients). Treated patients in the top $20 \%$ of the cost distribution accrued costs of over $\$ 7.0$ billion, which represented $69.0 \%$ of all costs accrued by the treated T2DM population (vs. $72.3 \%$ among all T2DM patients).

This study has several limitations common to most retrospective database studies. First, it was not possible to confirm diagnoses for T2DM, renal impairment, hypertension, or obesity. No laboratory data were available to further assess the level of renal impairment, and no information was available in the database regarding patients' height or weight. Thus, rates of obesity and renal impairment reported in the analysis are likely underestimated. Additionally, no information was available regarding blood glucose or glycated hemoglobin values, so the effect of glucose control on costs could not be assessed. Logistic regression model specifications were limited to the data available, and additional predictors of being an $\mathrm{HC}$ patient may exist (e.g., increased glycated hemoglobin value). Because this study used retrospective administrative claims, it was not feasible to assess the effect of an intervention (e.g., change in diabetes medication) on costs. Further, because our study used data from a managed care population, results may not be applicable to Medicaid, Medicare, or uninsured patients.

The goal of this study was to provide payers with a means of identifying patients who are at increased risk for becoming $\mathrm{HC}$, using real-world data. Once these patients are identified, personalized interventions could be developed that may decrease the likelihood of the patient becoming $\mathrm{HC}$. Interventions might include extra office visits for comorbid conditions, structured weight loss programs, or increased pharmacotherapy for glucose control. Economic evaluations to examine the cost-benefit structure of developing such interventions would be informative.

\section{Conclusions}

This study examined health care resource utilization and costs in a large, real-world, managed care population. In conclusion, it was found that patients with T2DM who make up the top $10 \%$ of a cost distribution for T2DM accrue, on average, 12 times more total annual health care costs than patients who make up the bottom $90 \%$ of the cost distribution. Further, T2DM patients who make up the top $20 \%$ of the cost distribution accrue, on average, 11 times more health care costs than patients who make up the bottom $80 \%$ of the cost distribution. Obesity and progression to insulin were found to predict the odds of being an $\mathrm{HC}$ patient and are two modifiable factors for T2DM patients. Further research is needed to explore potential interventions to reduce the likelihood 
that a patient becomes HC. Our study also found that cost of a hospitalization was the largest component of $\mathrm{HC}$ patients' total care costs. Reducing all-cause hospitalizations in patients with T2DM through interventions aimed at better management of T2DM (e.g., outpatient management, lifestyle changes) may help to reduce costs.

\section{Abbreviations}

CCl: Charlson Comorbidity Index; HC: High cost; ICD-9-CM: International Classification of Diseases, Ninth Revision, Clinical Modification; NHC: Not high cost; OR: Odds ratio; SD: Standard deviation; T2DM: Type 2 diabetes mellitus; US: United States; Cl: Confidence interval; ED: Emergency department; HMO: Health maintenance organization; OOP: Other outpatient; PPO: Preferred provider organization; SNF: Skilled nursing facility.

\section{Competing interests}

The authors declare that they have no competing interests.

\section{Authors' contributions}

JLM participated in the design of the study, carried out the research, performed the data analysis, and drafted the manuscript. SP conceived the study design, carried out the research, reviewed study results, and provided senior review of the manuscript. KB participated in the design of the study, carried out the research, reviewed study results, and provided senior review of the manuscript. JG participated in the design of the study, reviewed study results, and provided senior review of the manuscript. SDC participated in the design of the study, assisted with the data analysis, and helped to draft the manuscript. All authors read and approved the final manuscript.

\section{Authors' information}

JLM and SDC are employees of RTI Health Solutions, a research organization hired by AstraZeneca to conduct the analysis described in this manuscript. Neither JLM nor SDC have any competing interests to declare. SP is an employee of AstraZeneca. KB and JG are employees of Bristol-Myers Squibb.

\section{Acknowledgments}

This study was funded and conceived by AstraZeneca. The study design was developed in consultation with AstraZeneca, and results were reviewed by AstraZeneca. AstraZeneca did not influence the interpretation of results. The manuscript was reviewed by AstraZeneca prior to publication.

\section{Author details}

${ }^{1}$ RTI Health Solutions, 200 Park Offices Drive, Research Triangle Park, NC 27709, USA. ${ }^{2}$ AstraZeneca, 1800 Concord Pike, Wilmington, DE 19850, USA. ${ }^{3}$ Bristol-Myers Squibb, 777 Scudders Mill Road, Plainsboro, NJ 08536, USA.

Received: 7 March 2013 Accepted: 4 November 2013

Published: 27 February 2014

\section{References}

1. Centers for Disease Control and Prevention: National Diabetes Fact Sheet: National Estimates and General Information on Diabetes and Prediabetes in the United States, 2011. Atlanta: United States Department of Health and Human Services, Centers for Disease Control and Prevention; 2011.

2. American Heart Association: Heart Disease and Stroke Statistics-2008 Update. Dallas: American Heart Association; 2008.

3. National Institute of Diabetes and Digestive and Kidney Diseases: National Diabetes Statistics, 2007. NIH Publication No. 08-3892; 2008 [http://diabetes. niddk.nih.gov/dm/pubs/statistics/DM_Statistics.pdf]

4. American Diabetes Association: Standards of medical care in diabetes2011. Diabetes Care 2011, 34(Suppl 1):S11-S61.

5. Nathan DM, Buse JB, Davidson MB, Ferranni E, Holman RR, Sherwin R, Zinman B: Management of hyperglycemia in type 2 diabetes: a consensus algorithm for the initiation and adjustment of therapy. Diabetes Care 2009, 32:193-203.

6. American Diabetes Association: Economic costs of diabetes in the US in 2007. Diabetes Care 2008, 31(3):596-615

7. Bullano MF, Fisher MD, Grochulski WD, Meditto L, Willey VJ: Hypoglycemic events and glycosylated hemoglobin values in patients with type 2 diabetes mellitus newly initiated on insulin glargine or premixed insulin combination projects. Am J Health Syst Pharm 2006, 63(24):2473-2482.

8. Clarke PM, Glasziou P, Patel A, Chalmers J, Woodward M, Harrap SB, Salamon JA, ADVANCE Collaborative Group: Event rates, hospital utilization, and costs associated with major complications of diabetes: a multi-country comparative analysis. PLoS Med 2010, 7(2):e1000236.

9. Hammer M, Lammert M, Mejias SM, Kern W, Frier BM: Costs of managing severe hypoglycaemia in three European countries. J Med Econ 2009, 12(4):281-290

10. Lee WC, Balu S, Cobden D, Joshi AV, Pashos CL: Medication adherence and associated health-economic impact among patients with type 2 diabetes mellitus converting to insulin pen therapy: an analysis of third-party managed care claims data. Clin Ther 2006, 28(10):1712-1725. discussion 1710-1711.

11. Menzin J, Korn JR, Cohen J, Lobo F, Zhang B, Friedman M, Neumann PJ: Relationship between glycemic control and diabetes-related hospital costs in patients with type 1 or type 2 diabetes mellitus. J Manag Care Pharm 2010, 16(4):264-275.

12. Pelletier EM, Smith PJ, Boye KS, Misurski DA, Tunis SL, Minshall ME: Direct medical costs for type 2 diabetes mellitus complications in the US commercial payer setting: a resource for economic research. Appl Health Econ Health Policy 2008, 6(2-3):103-112.

13. Etemad LR, McCollam PL: Predictors of high-cost managed care patients with acute coronary syndrome. Curr Med Res Opin 2005, 21(12):1977-1984.

14. Conwell $L$, Cohen JW: Characteristics of people with high medical expenses in the U.S. civilian noninstitutionalized population, 2002. Statistical Brief \#73; 2005 [http://meps.ahrq.gov/mepsweb/data_files/publications/st73/stat73.pdf]

15. Charlson ME, Pompei P, Ales KL, MacKenzie CR: A new method of classifying prognostic comorbidity in longitudinal studies: development and validation. J Chronic Dis 1987, 40(5):373-383.

16. Olin GL, Machlin SR: Health Care Expenses in the Community Population, 1999, [MEPS Chartbook No. 11. AHRQ Pub. No. 03-0038.]. Rockville: Agency for Healthcare Research and Quality; 2003.

17. Hartmann J: Differences within high-cost patients: how do high-cost patients of different sexes and ages differ in their usage of health service sectors and their distribution of diagnoses? Sydney, Australia: Presentation at the International Health Economics Association; 2013.

18. Halanych JH, Safford MM, Keys WC, Person SD, Shikany JM, Kim YI, Centor $\mathrm{RM}$, Allison JJ: Burden of comorbid medical conditions and quality of diabetes care. Diabetes Care 2008, 30(12):2999-3004.

doi:10.1186/2049-3258-72-6

Cite this article as: Meyers et al:: The high-cost, type 2 diabetes mellitus patient: an analysis of managed care administrative data. Archives of Public Health 2014 72:6.

\section{Submit your next manuscript to BioMed Central and take full advantage of:}

- Convenient online submission

- Thorough peer review

- No space constraints or color figure charges

- Immediate publication on acceptance

- Inclusion in PubMed, CAS, Scopus and Google Scholar

- Research which is freely available for redistribution 